\title{
Fractional-order boundary value problems with Katugampola fractional integral conditions
}

\author{
Nazim I. Mahmudov* (1) and Sedef Emin
}

\author{
${ }^{*}$ Correspondence: \\ nazim.mahmudov@emu.edu.tr \\ Department of Mathematics, \\ Eastern Mediterranean University, \\ Gazimagusa, Turkish Republic of \\ Northern Cyprus
}

\begin{abstract}
In this paper, we study existence (uniqueness) of solutions for nonlinear fractional differential equations with Katugampola fractional integral conditions. Several fixed point theorems are used for sufficient conditions of existence (uniqueness) solutions of nonlinear differential equations such as Banach's contraction principle, the Leray-Schauder nonlinear alternative, and Krasnoselskii's fixed point theorem. Applications of the main results are also presented.
\end{abstract}

Keywords: Fractional differential equations; Katugampola derivative; Integral boundary conditions

\section{Introduction}

In recent years, boundary value problems for nonlinear fractional differential equations have been studied by several researchers. In fact, fractional differential equations have played an important role in physics, chemical technology, biology, economics, control theory, signal and image processing, see [1-25] and the references cited therein.

Boundary value problems of fractional differential equations and inclusions involve different kinds of boundary conditions such as nonlocal, integral, and multipoint boundary conditions. The fractional integral boundary conditions were introduced lately in [26] and nonlocal conditions were presented by Bitsadze, see [3].

In [4] authors gave sufficient criteria for existence of solutions for the following Caputo fractional differential equation:

$$
D^{q} x(t)=f(t, x(t)), \quad 0<t<T,
$$

subject to nonlocal generalized Riemann-Liouville fractional integral boundary conditions of the form

$$
\begin{aligned}
& x(0)=\gamma \frac{\rho^{1-\alpha}}{\Gamma(\alpha)} \int_{0}^{\xi} \frac{s^{\rho-1} x(s)}{\left(t^{\rho}-s^{\rho}\right)^{1-\alpha}} d s:=\gamma^{\rho} I^{\alpha} x(\xi), \\
& x(T)=\delta \frac{\rho^{1-\beta}}{\Gamma(\beta)} \int_{0}^{\varepsilon} \frac{s^{\rho-1} x(s)}{\left(\xi^{\rho}-s^{\rho}\right)^{1-\beta}} d s:=\delta^{\rho} I^{\beta} x(\varepsilon), \quad 0<\xi, \varepsilon<T,
\end{aligned}
$$


where $D^{q}$ denotes the Caputo fractional derivative of order $q,{ }^{\rho} I^{z}, z \in\{\alpha, \beta\}$, is the generalized Riemann-Liouville fractional integral of order $z>0, \rho>0, \xi, \varepsilon$ arbitrary, with $\xi, \varepsilon \in(0, T), \gamma, \delta \in R$ and $f:[0, T] \times R \rightarrow R$ is a continuous function.

In [5] authors established the existence of solutions for the following nonlinear RiemannLiouville fractional differential equation subject to nonlocal Erdelyi-Kober fractional integral conditions:

$$
\begin{aligned}
& D^{q} x(t)=f(t, x(t)), \quad t \in(0, T), \\
& x(0)=0, \quad \alpha x(T)=\sum_{i=1}^{m} \beta_{i} I_{\eta_{i}}^{\gamma_{i}, \delta_{i}} x\left(\xi_{i}\right),
\end{aligned}
$$

where $1<q \leq 2, D^{q}$ is the standard Riemann-Liouville fractional derivative of order $q, I_{\eta_{i}}^{\gamma_{i}, \delta_{i}}$ is the Erdelyi-Kober fractional integral of order $\delta_{i}>0$ with $\eta_{i}>0$ and $\gamma_{i} \in \mathbb{R}, i=1,2, \ldots, m$, $f:[0, T] \times \mathbb{R} \rightarrow \mathbb{R}$ is a continuous function and $\alpha, \beta_{i} \in \mathbb{R}, \xi_{i} \in(0, T), i=1,2, \ldots, m$, are given constants.

Motivated by the above papers, in this paper, we study the sufficient conditions of existence (uniqueness) solutions of nonlocal boundary conditions for the following nonlinear fractional differential equation of order $\alpha \in(2,3]$ :

$$
\left\{\begin{array}{l}
{ }^{c} D^{\alpha} x(t)=f(t, x(t)), \quad t \in[0, T], \\
x(T)=\beta^{\rho} I^{q} x(\epsilon), \quad 0<\epsilon<T, \\
x^{\prime}(T)=\gamma^{\rho} I^{q} x^{\prime}(\eta), \quad 0<\eta<T, \\
x^{\prime \prime}(T)=\delta^{\rho} I^{q} x^{\prime \prime}(\zeta), \quad 0<\zeta<T,
\end{array}\right.
$$

where $D^{\alpha}$ is the Caputo fractional derivative. ${ }^{\rho} I^{q}$ is the Katugampola integral of $q>0$, $\rho>0, f:[0, T] \times \mathbb{R} \rightarrow \mathbb{R}$ is a continuous function.

The rest of the paper is organized as follows. In Sect. 2, we recall some definitions and lemmas that we need in the sequel. In Sect. 3, several fixed point theorems are used to give sufficient conditions for existence (uniqueness) of solutions of (1) such as Banach's contraction principle, Krasnoselskii's fixed point theorem, and the Leray-Schauder nonlinear alternative. In Sect. 4, some illustrating examples are given.

\section{Preliminaries}

In this section, we recall some basic definitions of fractional calculus $[1,2,27]$ and some auxiliary lemmas which we need later.

Definition 1 ([1]) The Riemann-Liouville fractional integral of order $p>0$ of a continuous function $f:(0, \infty) \rightarrow \mathbb{R}$ is defined by

$$
J^{p} f(t)=\frac{1}{\Gamma(p)} \int_{0}^{t}(t-s)^{p-1} f(s) d s
$$

provided the right-hand side is point-wise defined on $(0, \infty)$, where $\Gamma$ is the gamma function defined by $\Gamma(p)=\int_{0}^{\infty} e^{-s} s^{p-1} d s$. 
Definition 2 ([1]) The Riemann-Liouville fractional derivative of order $p>0$ of a continuous function $f:(0, \infty) \rightarrow \mathbb{R}$ is defined by

$$
D_{0+}^{p} f(t)=\frac{1}{\Gamma(n-p)}\left(\frac{d}{d t}\right)^{n} \int_{0}^{t}(t-s)^{n-p-1} f(s) d s, \quad n-1<p<n
$$

where $n=[p]+1,[p]$ denotes the integer part of a real number $p$.

Definition 3 The Caputo derivative of order $p$ for a function $f:[0, \infty) \rightarrow \mathbb{R}$ can be written as

$$
{ }^{c} D^{p} f(t)=D_{0+}^{p}\left(f(t)-\sum_{k=0}^{n-1} \frac{t^{k}}{k !} f^{(k)}(0)\right), \quad t>0, n-1<p<n .
$$

Remark 4 If $f(t) \in C^{n}[0, \infty)$, then

$$
{ }^{c} D^{p} f(t)=\frac{1}{\Gamma(n-p)} \int_{0}^{t} \frac{f^{(n)}(s)}{(t-s)^{p+1-n}} d s=I^{n-p} f^{(n)}(t), \quad t>0, n-1<p<n .
$$

Definition 5 [28] Katugampola integral of order $q>0$ and $\rho>0$, of a function $f(t)$, for all $0<t<\infty$, is defined as

$$
{ }^{\rho} I^{q} f(t)=\frac{\rho^{1-q}}{\Gamma(q)} \int_{0}^{t} \frac{s^{\rho-1} f(s)}{\left(t^{\rho}-s^{\rho}\right)^{1-q}} d s
$$

provided the right-hand side is point-wise defined on $(0, \infty)$.

Remark 6 ([28]) The above definition corresponds to the one for Riemann-Liouville fractional integral of order $q>0$ when $\rho=1$, while the famous Hadamard fractional integral follows for $\rho \rightarrow 0$, that is,

$$
\lim _{\rho \rightarrow 0}{ }^{\rho} I^{q} f(t)=\frac{1}{\Gamma(q)} \int_{0}^{t}\left(\log \frac{t}{s}\right)^{q-1} \frac{f(s)}{s} d s
$$

Lemma 7 ([4]) Let $\rho, q>0$ and $p>0$ be the given constants. Then the following formula holds:

$$
{ }^{\rho} I^{q} t^{p}=\frac{\Gamma\left(\frac{p+\rho}{\rho}\right)}{\Gamma\left(\frac{p+\rho q+\rho}{\rho}\right)} \frac{t^{p+\rho q}}{\rho^{q}} .
$$

Lemma 8 ([1]) For $q>0$ and $x \in C(0, T) \cap L(0, T)$. Then the fractional differential equation ${ }^{c} D^{q} x(t)=0$ has a unique solution

$$
x(t)=c_{0}+c_{1} t+\cdots+c_{n-1} t^{n-1},
$$

and the following formula holds:

$$
I^{q} D^{q} x(t)=x(t)+c_{0}+c_{1} t+\cdots+c_{n-1} t^{n-1}
$$

where $c_{i} \in \mathbb{R}, i=0,1, \ldots, n-1$, and $n-1 \leq q<n$. 
Lemma 9 Let $2<\alpha \leq 3$ and $\beta, \gamma, \delta \in \mathbb{R}$. Then, for any $y \in C([0, T], \mathbb{R}), x$ is a solution of the following nonlinear fractional differential equation with Katugampola fractional integral conditions:

$$
\left\{\begin{array}{l}
{ }^{c} D^{\alpha} x(t)=y(t), \quad t \in[0, T], \\
x(T)=\beta^{\rho} I^{q} x(\epsilon), \quad 0<\epsilon<T, \\
x^{\prime}(T)=\gamma^{\rho} I^{q} x^{\prime}(\eta), \quad 0<\eta<T, \\
x^{\prime \prime}(T)=\delta^{\rho} I^{q} x^{\prime \prime}(\zeta), \quad 0<\zeta<T,
\end{array}\right.
$$

if and only if

$$
\begin{aligned}
x(t)= & J^{\alpha} y(t)+\frac{1}{\varpi_{1}(\beta, \epsilon)}\left(\beta^{\rho} I^{q} J^{\alpha} y(\epsilon)-J^{\alpha} y(T)\right) \\
& -\frac{1}{\varpi_{1}(\gamma, \eta)}\left(\frac{\varpi_{2}(\beta, \epsilon)}{\varpi_{1}(\beta, \epsilon)}-t\right)\left(\gamma^{\rho} I^{q} J^{\alpha-1} y(\eta)-J^{\alpha-1} y(T)\right) \\
& +\frac{1}{\varpi_{1}(\delta, \zeta)}\left(\frac{\varpi_{3}(\beta, \epsilon)}{2 \varpi_{1}(\beta, \epsilon)}-\frac{\varpi_{2}(\beta, \epsilon) \varpi_{2}(\gamma, \eta)}{\varpi_{1}(\beta, \epsilon) \varpi_{1}(\gamma, \eta)}\right. \\
& \left.+\frac{\varpi_{2}(\gamma, \eta) t}{\varpi_{1}(\gamma, \eta)}-\frac{t^{2}}{2}\right)\left(J^{\alpha-2} y(T)-\delta^{\rho} I^{q} J^{\alpha-2} y(\zeta)\right),
\end{aligned}
$$

where

$$
\begin{aligned}
& \varpi_{1}(\alpha, \xi)=\left(1-\alpha \frac{\xi^{\rho q}}{\rho^{q}} \frac{1}{\Gamma(q+1)}\right) \neq 0, \\
& \varpi_{2}(\alpha, \xi)=\left(T-\alpha \frac{\xi^{\rho q+1}}{\rho^{q}} \frac{\Gamma\left(\frac{1+\rho}{\rho}\right)}{\Gamma\left(\frac{1+\rho q+\rho}{\rho}\right)}\right), \\
& \varpi_{3}(\alpha, \xi)=\left(T^{2}-\alpha \frac{\xi^{\rho q+2}}{\rho^{q}} \frac{\Gamma\left(\frac{2+\rho}{\rho}\right)}{\Gamma\left(\frac{2+\rho q+\rho}{\rho}\right)}\right) .
\end{aligned}
$$

Proof Using Lemma 8, the general solution of the nonlinear fractional differential equation in (2) can be represented as

$$
x(t)=c_{0}+c_{1} t+c_{2} t^{2}+J^{\alpha} y(t), \quad c_{0}, c_{1}, c_{2} \in \mathbb{R} .
$$

By using the first integral condition of problem (2) and applying the Katugampola integral on (7), we obtain

$$
\begin{aligned}
c_{0}+ & c_{1} T+c_{2} T^{2}+J^{\alpha} y(T) \\
= & \beta c_{0} \frac{\epsilon^{\rho q}}{\rho^{q}} \frac{1}{\Gamma(q+1)}+\beta c_{1} \frac{\epsilon^{\rho q+1}}{\rho^{q}} \frac{\Gamma\left(\frac{1+\rho}{\rho}\right)}{\Gamma\left(\frac{1+\rho q+\rho}{\rho}\right)} \\
& +\beta c_{2} \frac{\epsilon^{\rho q+2}}{\rho^{q}} \frac{\Gamma\left(\frac{2+\rho}{\rho}\right)}{\Gamma\left(\frac{2+\rho q+\rho}{\rho}\right)}+\beta^{\rho} I^{q} J^{\alpha} y(\epsilon) .
\end{aligned}
$$


After collecting the similar terms in one part, we have the following equation:

$$
\begin{aligned}
c_{0}\left(1-\beta \frac{\epsilon^{\rho q}}{\rho^{q}} \frac{1}{\Gamma(q+1)}\right)+c_{1}\left(T-\beta c_{1} \frac{\epsilon^{\rho q+1}}{\rho^{q}} \frac{\Gamma\left(\frac{1+\rho}{\rho}\right)}{\Gamma\left(\frac{1+\rho q+\rho}{\rho}\right)}\right) \\
\quad+c_{2}\left(T^{2}-\beta c_{2} \frac{\epsilon^{\rho q+2}}{\rho^{q}} \frac{\Gamma\left(\frac{2+\rho}{\rho}\right)}{\Gamma\left(\frac{2+\rho q+\rho}{\rho}\right)}\right) \\
=\beta^{\rho} I^{q} J^{\alpha} y(\epsilon)-J^{\alpha} y(T) .
\end{aligned}
$$

Rewriting equation (8) by using (4), (5), and (6), we obtain

$$
c_{0} \varpi_{1}(\beta, \epsilon)+c_{1} \varpi_{2}(\beta, \epsilon)+c_{2} \varpi_{3}(\beta, \epsilon)=\beta^{\rho} I^{q} J^{\alpha} y(\epsilon)-J^{\alpha} y(T) .
$$

Then, taking the derivative of (7) and using the second integral condition of (2), we get

$$
x^{\prime}(T)=c_{1}+2 c_{2} T+J^{\alpha-1} y(T)
$$

Now, applying the Katugampola integral on (10), we have

$$
\begin{aligned}
c_{1} & +2 c_{2} T+J^{\alpha-1} y(T) \\
= & \gamma c_{1} \frac{\eta^{\rho q}}{\rho^{q}} \frac{1}{\Gamma(q+1)} \\
& +2 c_{2} \gamma \frac{\eta^{\rho q+1}}{\rho^{q}} \frac{\Gamma\left(\frac{1+\rho}{\rho}\right)}{\Gamma\left(\frac{1+\rho q+\rho}{\rho}\right)}+\gamma^{\rho} I^{q} J^{\alpha-1} y(\eta) .
\end{aligned}
$$

The above equation (11) implies that

$$
\begin{aligned}
& c_{1}\left(1-\gamma \frac{\eta^{\rho q}}{\rho^{q}} \frac{1}{\Gamma(q+1)}\right)+2 c_{2}\left(T-\gamma \frac{\eta^{\rho q+1}}{\rho^{q}} \frac{\Gamma\left(\frac{1+\rho}{\rho}\right)}{\Gamma\left(\frac{1+\rho q+\rho}{\rho}\right)}\right) \\
& \quad=\gamma^{\rho} I^{q} J^{\alpha-1} y(\eta)-J^{\alpha-1} y(T) .
\end{aligned}
$$

Also, by using (4) and (5), equation (12) can be written as

$$
\begin{aligned}
& c_{1} \varpi_{1}(\gamma, \eta)+2 c_{2} \varpi_{2}(\gamma, \eta) \\
& \quad=\gamma^{\rho} I^{q} J^{\alpha-1} y(\eta)-J^{\alpha-1} y(T) .
\end{aligned}
$$

By using the last integral condition of (2) and applying Katugampola integral operator on the second derivative of (10), we have

$$
2 c_{2}+J^{\alpha-2} y(T)=2 \delta c_{2} \frac{\zeta^{\rho q}}{\rho^{q}} \frac{1}{\Gamma(q+1)}+\delta^{\rho} I^{q} J^{\alpha-2} y(\zeta) .
$$

Hence, we obtain the following equation:

$$
2 c_{2}\left(1-\delta \frac{\zeta^{\rho q}}{\rho^{q}} \frac{1}{\Gamma(q+1)}\right)=\delta^{\rho} I^{q} J^{\alpha-2} y(\zeta)-J^{\alpha-2} y(T)
$$


By using (4), equation (14) can be written as

$$
2 c_{2} \varpi_{1}(\delta, \zeta)=\delta^{\rho} I^{q} J^{\alpha-2} y(\zeta)-J^{\alpha-2} y(T)
$$

Moreover, equation (15) implies that

$$
c_{2}=\frac{1}{2 \varpi_{1}(\delta, \zeta)}\left(\delta^{\rho} I^{q} J^{\alpha-2} y(\zeta)-J^{\alpha-2} y(T)\right)
$$

Substituting the values of (16) in (13), we get

$$
\begin{aligned}
c_{1}= & \frac{1}{\varpi_{1}(\gamma, \eta)}\left(\gamma^{\rho} I^{q} J^{\alpha-1} y(\eta)-J^{\alpha-1} y(T)\right) \\
& -\frac{\varpi_{2}(\gamma, \eta)}{\varpi_{1}(\gamma, \eta) \varpi_{1}(\delta, \zeta)}\left(\delta^{\rho} I^{q} J^{\alpha-2} y(\zeta)-J^{\alpha-2} y(T)\right) .
\end{aligned}
$$

Now, substituting the values of (16) and (17) in (9), we obtain

$$
\begin{aligned}
c_{0}= & \frac{1}{\varpi_{1}(\beta, \epsilon)}\left(\beta^{\rho} I^{q} J^{\alpha} y(\epsilon)-J^{\alpha} y(T)\right) \\
& -\frac{\varpi_{2}(\beta, \epsilon)}{\varpi_{1}(\beta, \epsilon) \varpi_{1}(\gamma, \eta)}\left(\gamma^{\rho} I^{q} J^{\alpha-1} y(\eta)-J^{\alpha-1} y(T)\right) \\
& -\frac{\varpi_{3}(\beta, \epsilon)}{2 \varpi_{1}(\beta, \epsilon) \varpi_{1}(\delta, \zeta)}\left(\delta^{\rho} I^{q} J^{\alpha-2} y(\zeta)-J^{\alpha-2} y(T)\right) \\
& +\frac{\varpi_{2}(\beta, \epsilon) \varpi_{2}(\gamma, \eta)}{\varpi_{1}(\beta, \epsilon) \varpi_{1}(\gamma, \eta) \varpi_{1}(\delta, \zeta)}\left(\delta^{\rho} I^{q} J^{\alpha-2} y(\zeta)-J^{\alpha-2} y(T)\right) .
\end{aligned}
$$

Finally, substituting the values of (18), (17), and (16) in equation (7), we obtain the general solution of problem (2) which is (3). Converse is also true by using the direct computation.

\section{Main results}

Let us denote by $C=C([0, T], \mathbb{R})$ the Banach space of all continuous functions from $[0, T] \rightarrow \mathbb{R}$ endowed with a topology of uniform convergence with the norm defined by $\|x\|=\sup \{|x(t)|: t \in[0, T]\}$.

We define an operator $H: C \rightarrow C$ on problem (1) as

$$
\begin{aligned}
(H x)(t)= & J^{\alpha} f(s, x(s))(t)+\frac{1}{\varpi_{1}(\beta, \epsilon)}\left(\beta^{\rho} I^{q} J^{\alpha} f(s, x(s))(\epsilon)\right. \\
& \left.-J^{\alpha} f(s, x(s))(T)\right)-\frac{1}{\varpi_{1}(\gamma, \eta)}\left(\frac{\varpi_{2}(\beta, \epsilon)}{\varpi_{1}(\beta, \epsilon)}-t\right) \\
& \times\left(\gamma^{\rho} I^{q} J^{\alpha-1} f(s, x(s))(\eta)-J^{\alpha-1} f(s, x(s))(T)\right) \\
& +\frac{1}{\varpi_{1}(\delta, \zeta)}\left(\frac{\varpi_{3}(\beta, \epsilon)}{2 \varpi_{1}(\beta, \epsilon)}-\frac{\varpi_{2}(\beta, \epsilon) \varpi_{2}(\gamma, \eta)}{\varpi_{1}(\beta, \epsilon) \varpi_{1}(\gamma, \eta)}\right. \\
& \left.+\frac{\varpi_{2}(\gamma, \eta) t}{\varpi_{1}(\gamma, \eta)}-\frac{t^{2}}{2}\right)\left(J^{\alpha-2} f(s, x(s))(T)\right. \\
& \left.-\delta^{\rho} I^{q} J^{\alpha-2} f(s, x(s))(\zeta)\right) .
\end{aligned}
$$


Also, we define the notations

$$
\begin{aligned}
\Phi= & \frac{T^{\alpha}}{\Gamma(\alpha+1)}+\frac{1}{\left|\varpi_{1}(\beta, \epsilon)\right| \Gamma(\alpha+1)} \\
& \times\left(|\beta| \frac{\Gamma\left(\frac{\alpha+\rho}{\rho}\right)}{\Gamma\left(\frac{\alpha+\rho q+\rho}{\rho}\right)} \frac{\epsilon^{\alpha+\rho q}}{\rho^{q}}+T^{\alpha}\right) \\
& +\frac{1}{\left|\varpi_{1}(\gamma, \eta)\right| \Gamma(\alpha)}\left(\frac{\left|\varpi_{2}(\beta, \epsilon)\right|}{\left|\varpi_{1}(\beta, \epsilon)\right|}+T\right) \\
& \times\left(|\gamma| \frac{\Gamma\left(\frac{\alpha-1+\rho}{\rho}\right)}{\Gamma\left(\frac{\alpha-1+\rho q+\rho}{\rho}\right)} \frac{\eta^{\alpha-1+\rho q}}{\rho^{q}}+T^{\alpha-1}\right) \\
& +\frac{1}{\left|\varpi_{1}(\delta, \zeta)\right| \Gamma(\alpha-1)}\left(\frac{\left|\varpi_{3}(\beta, \epsilon)\right|}{2\left|\varpi_{1}(\beta, \epsilon)\right|}\right. \\
& \left.+\frac{\left|\varpi_{2}(\beta, \epsilon)\right|\left|\varpi_{2}(\gamma, \eta)\right|}{\left|\varpi_{1}(\beta, \epsilon)\right|\left|\varpi_{1}(\gamma, \eta)\right|}+\frac{\left|\varpi_{2}(\gamma, \eta)\right| T}{\left|\varpi_{1}(\gamma, \eta)\right|}+\frac{T^{2}}{2}\right) \\
& \times\left(|\delta| \frac{\Gamma\left(\frac{\alpha-2+\rho}{\rho}\right)}{\Gamma\left(\frac{\alpha-2+\rho q+\rho}{\rho}\right)} \frac{\zeta^{\alpha-2+\rho q}}{\rho^{q}}+T^{\alpha-2}\right)
\end{aligned}
$$

and

$$
\begin{aligned}
\Phi_{1}= & \frac{|\beta|}{\left|\varpi_{1}(\beta, \epsilon)\right| \Gamma(\alpha+1)} \frac{\Gamma\left(\frac{\alpha+\rho}{\rho}\right)}{\Gamma\left(\frac{\alpha+\rho q+\rho}{\rho}\right)} \frac{\epsilon^{\alpha+\rho q}}{\rho^{q}} \\
& +\frac{|\gamma|}{\left|\varpi_{1}(\gamma, \eta)\right| \Gamma(\alpha)}\left(\frac{\left|\varpi_{2}(\beta, \epsilon)\right|}{\left|\varpi_{1}(\beta, \epsilon)\right|}+T\right) \frac{\Gamma\left(\frac{\alpha-1+\rho}{\rho}\right)}{\Gamma\left(\frac{\alpha-1+\rho q+\rho}{\rho}\right)} \frac{\eta^{\alpha-1+\rho q}}{\rho^{q}} \\
& +\frac{|\delta|}{\left|\varpi_{1}(\delta, \zeta)\right| \Gamma(\alpha-1)}\left(\frac{\left|\varpi_{3}(\beta, \epsilon)\right|}{2\left|\varpi_{1}(\beta, \epsilon)\right|}+\frac{\left|\varpi_{2}(\beta, \epsilon)\right|\left|\varpi_{2}(\gamma, \eta)\right|}{\left|\varpi_{1}(\beta, \epsilon)\right|\left|\varpi_{1}(\gamma, \eta)\right|}\right. \\
& \left.+\frac{\left|\varpi_{2}(\gamma, \eta)\right| T}{\left|\varpi_{1}(\gamma, \eta)\right|}+\frac{T^{2}}{2}\right) \frac{\Gamma\left(\frac{\alpha-2+\rho}{\rho}\right)}{\Gamma\left(\frac{\alpha-2+\rho q+\rho}{\rho}\right)} \frac{\zeta^{\alpha-2+\rho q}}{\rho^{q}} .
\end{aligned}
$$

In the following subsections, we prove existence (uniqueness) results for the boundary value problem (1) by using Banach's fixed point theorem, the Leray-Schauder nonlinear alternative, and Krasnoselskii's fixed point theorem.

\subsection{Existence and uniqueness result}

Theorem 10 Let $f:[0, T] \times \mathbb{R} \rightarrow \mathbb{R}$ be a continuous function. Assume that:

$\left(S_{1}\right)|f(t, x)-f(t, y)| \leq L\|x-y\|$ for all $t \in[0, T], L>0, x, y \in \mathbb{R}$;

$\left(S_{2}\right) L \Phi<1$, where $\Phi$ is defined by $(20)$.

Then the boundary value problem (1) has a unique solution on $[0, T]$.

Proof By using the operator $H$, which is defined by (19), we obtain

$$
\begin{aligned}
|(H x)(t)-(H y)(t)| \leq & J^{\alpha}|f(s, x(s))-f(s, y(s))|(T) \\
& +\frac{|\beta|}{\left|\varpi_{1}(\beta, \epsilon)\right|} \rho^{q} I^{\alpha} J^{\alpha} f(s, x(s))-f(s, y(s)) \mid(\epsilon)
\end{aligned}
$$




$$
\begin{aligned}
& +\frac{1}{\left|\varpi_{1}(\beta, \epsilon)\right|} J^{\alpha}|f(s, x(s))-f(s, y(s))|(T) \\
& +\frac{|\gamma|}{\left|\varpi_{1}(\gamma, \eta)\right|}\left(\frac{\left|\varpi_{2}(\beta, \epsilon)\right|}{\left|\varpi_{1}(\beta, \epsilon)\right|}+T\right) \\
& \times{ }^{\rho} I^{q} J^{\alpha-1}|f(s, x(s))-f(s, y(s))|(\eta) \\
& +\frac{1}{\left|\varpi_{1}(\gamma, \eta)\right|}\left(\frac{\left|\varpi_{2}(\beta, \epsilon)\right|}{\left|\varpi_{1}(\beta, \epsilon)\right|}+T\right) \\
& \times J^{\alpha-1}|f(s, x(s))-f(s, y(s))|(T) \\
& +\frac{1}{\left|\varpi_{1}(\delta, \zeta)\right|}\left(\frac{\left|\varpi_{3}(\beta, \epsilon)\right|}{2\left|\varpi_{1}(\beta, \epsilon)\right|}+\frac{\left|\varpi_{2}(\beta, \epsilon)\right|\left|\varpi_{2}(\gamma, \eta)\right|}{\left|\varpi_{1}(\beta, \epsilon)\right|\left|\varpi_{1}(\gamma, \eta)\right|}\right. \\
& \left.+\frac{\left|\varpi_{2}(\gamma, \eta)\right| T}{\left|\varpi_{1}(\gamma, \eta)\right|}+\frac{T^{2}}{2}\right) \\
& \times J^{\alpha-2}|f(s, x(s))-f(s, y(s))|(T) \\
& +\frac{|\delta|}{\left|\varpi_{1}(\delta, \zeta)\right|}\left(\frac{\left|\varpi_{3}(\beta, \epsilon)\right|}{2\left|\varpi_{1}(\beta, \epsilon)\right|}+\frac{\left|\varpi_{2}(\beta, \epsilon)\right|\left|\varpi_{2}(\gamma, \eta)\right|}{\left|\varpi_{1}(\beta, \epsilon)\right|\left|\varpi_{1}(\gamma, \eta)\right|}\right. \\
& \left.+\frac{\left|\varpi_{2}(\gamma, \eta)\right| T}{\left|\varpi_{1}(\gamma, \eta)\right|}+\frac{T^{2}}{2}\right) \\
& \times{ }^{\rho} I^{q} J^{\alpha-2}|f(s, x(s))-f(s, y(s))|(\zeta) \\
& \leq L\|x-y\|\left\{J^{\alpha}(1)(T)+\frac{1}{\left|\varpi_{1}(\beta, \epsilon)\right|}\left(|\beta|^{\rho} I^{q} J^{\alpha}(1)(\epsilon)\right.\right. \\
& \left.+J^{\alpha}(1)(T)\right) \\
& +\frac{1}{\left|\varpi_{1}(\gamma, \eta)\right|}\left(\frac{\left|\varpi_{2}(\beta, \epsilon)\right|}{\left|\varpi_{1}(\beta, \epsilon)\right|}+T\right)\left(|\gamma|^{\rho} I^{q} J^{\alpha-1}(1)(\eta)\right. \\
& \left.+J^{\alpha-1}(1)(T)\right) \\
& +\frac{1}{\left|\varpi_{1}(\delta, \zeta)\right|}\left(\frac{\left|\varpi_{3}(\beta, \epsilon)\right|}{2\left|\varpi_{1}(\beta, \epsilon)\right|}+\frac{\left|\varpi_{2}(\beta, \epsilon)\right|\left|\varpi_{2}(\gamma, \eta)\right|}{\left|\varpi_{1}(\beta, \epsilon)\right|\left|\varpi_{1}(\gamma, \eta)\right|}\right. \\
& \left.+\frac{\left|\varpi_{2}(\gamma, \eta)\right| T}{\left|\varpi_{1}(\gamma, \eta)\right|}+\frac{T^{2}}{2}\right)\left(|\delta|^{\rho} I^{q} J^{\alpha-2}(1)(\zeta)\right. \\
& \left.\left.+J^{\alpha-2}(1)(T)\right)\right\} \\
& \leq L\left\{\frac{T^{\alpha}}{\Gamma(\alpha+1)}+\frac{1}{\left|\varpi_{1}(\beta, \epsilon)\right| \Gamma(\alpha+1)}\right. \\
& \times\left(|\beta| \frac{\Gamma\left(\frac{\alpha+\rho}{\rho}\right)}{\Gamma\left(\frac{\alpha+\rho q+\rho}{\rho}\right)} \frac{\epsilon^{\alpha+\rho q}}{\rho^{q}}+T^{\alpha}\right) \\
& +\frac{1}{\left|\varpi_{1}(\gamma, \eta)\right| \Gamma(\alpha)}\left(\frac{\left|\varpi_{2}(\beta, \epsilon)\right|}{\left|\varpi_{1}(\beta, \epsilon)\right|}+T\right) \\
& \times\left(|\gamma| \frac{\Gamma\left(\frac{\alpha-1+\rho}{\rho}\right)}{\Gamma\left(\frac{\alpha-1+\rho q+\rho}{\rho}\right)} \frac{\eta^{\alpha-1+\rho q}}{\rho^{q}}+T^{\alpha-1}\right) \\
& +\frac{1}{\left|\varpi_{1}(\delta, \zeta)\right| \Gamma(\alpha-1)}\left(\frac{\left|\varpi_{3}(\beta, \epsilon)\right|}{2\left|\varpi_{1}(\beta, \epsilon)\right|}\right.
\end{aligned}
$$




$$
\begin{aligned}
& \left.+\frac{\left|\varpi_{2}(\beta, \epsilon)\right|\left|\varpi_{2}(\gamma, \eta)\right|}{\left|\varpi_{1}(\beta, \epsilon)\right|\left|\varpi_{1}(\gamma, \eta)\right|}+\frac{\left|\varpi_{2}(\gamma, \eta)\right| T}{\left|\varpi_{1}(\gamma, \eta)\right|}+\frac{T^{2}}{2}\right) \\
& \left.\quad \times\left(|\delta| \frac{\Gamma\left(\frac{\alpha-2+\rho}{\rho}\right)}{\Gamma\left(\frac{\alpha-2+\rho q+\rho}{\rho}\right)} \frac{\zeta^{\alpha-2+\rho q}}{\rho^{q}}+T^{\alpha-2}\right)\right\}\|x-y\| \\
& =L \Phi\|x-y\|
\end{aligned}
$$

for any $x, y \in C$ and for each $t \in[0, T]$. This implies that $\|H x-H y\| \leq L \Phi\|x-y\|$. As $L \Phi<1$, the operator $H: C \rightarrow C$ is a contraction mapping. As a result, the boundary value problem (1) has a unique solution on $[0, T]$.

\subsection{Existence results}

Lemma 11 Let $E$ be a Banach space, $C$ be a closed, convex subset of $E, U$ be an open subset of $C$ and $0 \in U$. Suppose that $A: \bar{U} \rightarrow C$ is a continuous, compact map. Then either

(i) A has a fixed point in $\bar{U}$, or

(ii) there are $x \in \partial U$ (the boundary of $U$ in $C$ ) and $\mu \in(0,1)$ with $x=\mu A(x)$.

Theorem 12 Let $f:[0, T] \times \mathbb{R} \rightarrow \mathbb{R}$ be a continuous function. Assume that:

$\left(S_{3}\right)$ There exist a nonnegative function $\Omega \in C([0, T], \mathbb{R})$ and a nondecreasing function $\Psi:[0, \infty) \rightarrow(0, \infty)$ such that

$$
|f(t, u)| \leq \Omega(t) \Psi(|u|) \quad \text { for any }(t, u) \in[0, T] \times \mathbb{R}
$$

$\left(S_{4}\right)$ There exists a constant $M>0$ such that

$$
\frac{M}{\Psi(M)\|\Omega\| \Phi}>1
$$

where $\Phi$ in (20).

Then problem (1) has at least one solution on $[0, T]$.

Proof Let $B_{d}=\{x \in C:\|x\| \leq d\}$ be a closed bounded subset in $C([0, T], \mathbb{R})$. Notice that problem (1) is equivalent to the problem of finding a fixed point of $H$.

As a first step, we show that the operator $H$, which is defined by (19), maps bounded sets into bounded sets in $C([0, T], \mathbb{R})$. Then, for $t \in[0, T]$, we have

$$
\begin{aligned}
|H(x)(t)| \leq & J^{\alpha}|f(s, x(s))|(T) \\
& +\frac{1}{\left|\varpi_{1}(\beta, \epsilon)\right|}\left(|\beta|^{\rho} I^{q} J^{\alpha}|f(s, x(s))|(\epsilon)\right. \\
& \left.+J^{\alpha}|f(s, x(s))|(T)\right) \\
& +\frac{1}{\left|\varpi_{1}(\gamma, \eta)\right|}\left(\frac{\left|\varpi_{2}(\beta, \epsilon)\right|}{\left|\varpi_{1}(\beta, \epsilon)\right|}+T\right) \\
& \times\left(|\gamma| J^{\alpha-1}|f(s, x(s))|(\eta)+J^{\alpha-1}|f(s, x(s))|(T)\right) \\
& +\frac{1}{\left|\varpi_{1}(\delta, \zeta)\right|}\left(\frac{\left|\varpi_{3}(\beta, \epsilon)\right|}{2\left|\varpi_{1}(\beta, \epsilon)\right|}+\frac{\left|\varpi_{2}(\beta, \epsilon)\right|\left|\varpi_{2}(\gamma, \eta)\right|}{\left|\varpi_{1}(\beta, \epsilon)\right|\left|\varpi_{1}(\gamma, \eta)\right|}\right. \\
& \left.+\frac{\left|\varpi_{2}(\gamma, \eta)\right| T}{\left|\varpi_{1}(\gamma, \eta)\right|}+\frac{T^{2}}{2}\right)
\end{aligned}
$$




$$
\begin{aligned}
& \times\left(J^{\alpha-2}|f(s, x(s))|(T)+|\delta|^{\rho} I^{q} J^{\alpha-2}|f(s, x(s))|(\zeta)\right) \\
& \leq \Psi(\|x\|) J^{\alpha} \Omega(s)(T) \\
& +\frac{\Psi(\|x\|)}{\left|\varpi_{1}(\beta, \epsilon)\right|}\left(|\beta|^{\rho} I^{q} J^{\alpha} \Omega(s)(\epsilon)\right. \\
& \left.+J^{\alpha} \Omega(s)(T)\right) \\
& +\frac{\Psi(\|x\|)}{\left|\varpi_{1}(\gamma, \eta)\right|}\left(\frac{\left|\varpi_{2}(\beta, \epsilon)\right|}{\left|\varpi_{1}(\beta, \epsilon)\right|}+T\right) \\
& \times\left(|\gamma|^{\rho} I^{q} J^{\alpha-1} \Omega(s)(\eta)+J^{\alpha-1} \Omega(s)(T)\right) \\
& +\frac{\Psi(\|x\|)}{\left|\varpi_{1}(\delta, \zeta)\right|}\left(\frac{\left|\varpi_{3}(\beta, \epsilon)\right|}{2\left|\varpi_{1}(\beta, \epsilon)\right|}+\frac{\left|\varpi_{2}(\beta, \epsilon)\right|\left|\varpi_{2}(\gamma, \eta)\right|}{\left|\varpi_{1}(\beta, \epsilon)\right|\left|\varpi_{1}(\gamma, \eta)\right|}\right. \\
& \left.+\frac{\left|\varpi_{2}(\gamma, \eta)\right| T}{\left|\varpi_{1}(\gamma, \eta)\right|}+\frac{T^{2}}{2}\right) \\
& \times\left(J^{\alpha-2} \Omega(s)(T)+|\delta|^{\rho} I^{q} J^{\alpha-2} \Omega(s)(\zeta)\right) \\
& \leq\|\Omega\| \Psi(d)\left\{\frac{T^{\alpha}}{\Gamma(\alpha+1)}+\frac{1}{\left|\varpi_{1}(\beta, \epsilon)\right| \Gamma(\alpha+1)}\right. \\
& \times\left(|\beta| \frac{\Gamma\left(\frac{\alpha+\rho}{\rho}\right)}{\Gamma\left(\frac{\alpha+\rho q+\rho}{\rho}\right)} \frac{\epsilon^{\alpha+\rho q}}{\rho^{q}}+T^{\alpha}\right) \\
& +\frac{1}{\left|\varpi_{1}(\gamma, \eta)\right| \Gamma(\alpha)}\left(\frac{\left|\varpi_{2}(\beta, \epsilon)\right|}{\left|\varpi_{1}(\beta, \epsilon)\right|}+T\right) \\
& \times\left(|\gamma| \frac{\Gamma\left(\frac{\alpha-1+\rho}{\rho}\right)}{\Gamma\left(\frac{\alpha-1+\rho q+\rho}{\rho}\right)} \frac{\eta^{\alpha-1+\rho q}}{\rho^{q}}+T^{\alpha-1}\right) \\
& +\frac{1}{\left|\varpi_{1}(\delta, \zeta)\right| \Gamma(\alpha-1)}\left(\frac{\left|\varpi_{3}(\beta, \epsilon)\right|}{2\left|\varpi_{1}(\beta, \epsilon)\right|}\right. \\
& \left.+\frac{\left|\varpi_{2}(\beta, \epsilon)\right|\left|\varpi_{2}(\gamma, \eta)\right|}{\left|\varpi_{1}(\beta, \epsilon)\right|\left|\varpi_{1}(\gamma, \eta)\right|}+\frac{\left|\varpi_{2}(\gamma, \eta)\right| T}{\left|\varpi_{1}(\gamma, \eta)\right|}+\frac{T^{2}}{2}\right) \\
& \left.\times\left(|\delta| \frac{\Gamma\left(\frac{\alpha-2+\rho}{\rho}\right)}{\Gamma\left(\frac{\alpha-2+\rho q+\rho}{\rho}\right)} \frac{\zeta^{\alpha-2+\rho q}}{\rho^{q}}+T^{\alpha-2}\right)\right\} \\
& =\|\Omega\| \Psi(d) \Phi,
\end{aligned}
$$

which leads to $\|H(x)\| \leq\|\Omega\| \Psi(d) \Phi$. By $\left(S_{4}\right)$ there exists $d>0$ such that $\Psi(d)\|\Omega\| \Phi<d$.

Next, we show that the map $H: C([0, T], \mathbb{R}) \rightarrow C([0, T], \mathbb{R})$ is completely continuous. Therefore, to prove that the map $H$ is completely continuous, we show that $H$ is a map from bounded sets into equicontinuous sets of $C([0, T], \mathbb{R})$. Let us choose $t_{1}, t_{2}$ from the interval $[0, T]$ and also $t_{1}<t_{2}$. Then we have

$$
\begin{aligned}
& \left|(H x)\left(t_{2}\right)-(H x)\left(t_{1}\right)\right| \\
& \leq\left|J^{\alpha} f(s, x(s))\left(t_{2}\right)-J^{\alpha} f(s, x(s))\left(t_{1}\right)\right| \\
& \quad+\frac{\left|t_{2}-t_{1}\right|}{\left|\varpi_{1}(\gamma, \eta)\right|}\left(|\gamma|^{q} J^{\alpha-1}|f(s, x(s))|(\eta)+J^{\alpha-1}|f(s, x(s))|(T)\right) \\
& \quad+\frac{1}{\left|\varpi_{1}(\delta, \zeta)\right|}\left(\frac{\left|\varpi_{2}(\gamma, \eta)\right|}{\left|\varpi_{1}(\gamma, \eta)\right|}\left|t_{2}-t_{1}\right|+\frac{\left|t_{2}^{2}-t_{1}^{2}\right|}{2}\right)
\end{aligned}
$$




$$
\begin{aligned}
& \times\left(J^{\alpha-2}|f(s, x(s))|(T)+|\delta|^{\rho} I^{q} J^{\alpha-2}|f(s, x(s))|(\zeta)\right) \\
\leq & \frac{\Psi(\|x\|) \Omega(s)}{\Gamma(\alpha)}\left[\int_{0}^{t_{1}}\left[\left(t_{2}-s\right)^{\alpha-1}-\left(t_{1}-s\right)^{\alpha-1}\right] d s+\int_{t_{1}}^{t_{2}}\left(t_{2}-s\right)^{\alpha-1} d s\right] \\
& +\left|t_{2}-t_{1}\right| \frac{\Psi(\|x\|)}{\left|\varpi_{1}(\gamma, \eta)\right|}\left(|\gamma| J^{\alpha-1} \Omega(s)(\eta)+J^{\alpha-1} \Omega(s)(T)\right. \\
& \left.+\left(\frac{\left|\varpi_{2}(\gamma, \eta)\right|}{\left|\varpi_{1}(\delta, \zeta)\right|}+\frac{\left|t_{2}+t_{1}\right|}{2} \frac{\left|\varpi_{1}(\gamma, \eta)\right|}{\left|\varpi_{1}(\delta, \zeta)\right|}\right)\left(J^{\alpha-2} \Omega(s)(T)+|\delta|^{\rho} I^{q} J^{\alpha-2} \Omega(s)(\zeta)\right)\right) \\
\leq & \frac{\Psi(d)\|\Omega\| \mid}{\Gamma(\alpha)}\left[\int_{0}^{t_{1}}\left[\left(t_{2}-s\right)^{\alpha-1}-\left(t_{1}-s\right)^{\alpha-1}\right] d s+\int_{t_{1}}^{t_{2}}\left(t_{2}-s\right)^{\alpha-1} d s\right] \\
& +\left|t_{2}-t_{1}\right| \frac{\Psi(d)\|\Omega\|}{\left|\varpi_{1}(\gamma, \eta)\right|}\left\{\frac{1}{\Gamma(\alpha)}\left(T^{\alpha-1}+|\gamma| \frac{\Gamma\left(\frac{\alpha-1+\rho}{\rho}\right)}{\Gamma\left(\frac{\alpha-1+\rho q+\rho}{\rho}\right)} \frac{\eta^{\alpha-1+\rho q}}{\rho^{q}}\right)\right. \\
& +\frac{1}{\Gamma(\alpha-1)}\left(\frac{\left|\varpi_{2}(\gamma, \eta)\right|}{\left|\varpi_{1}(\delta, \zeta)\right|}+\frac{\left|t_{2}+t_{1}\right|}{2} \frac{\left|\varpi_{1}(\gamma, \eta)\right|}{\left|\varpi_{1}(\delta, \zeta)\right|}\right) \\
& \left.\times\left(T^{\alpha-2}+|\delta| \frac{\Gamma\left(\frac{\alpha-2+\rho}{\rho}\right)}{\Gamma\left(\frac{\alpha-2+\rho q+\rho}{\rho}\right)} \frac{\zeta^{\alpha-2+\rho q}}{\rho^{q}}\right)\right\} .
\end{aligned}
$$

It is clear that the right-hand side of (22) is independent of $x$. Therefore, as $t_{2}-t_{1} \rightarrow 0$, inequality (22) tends to zero. That means $H$ is equicontinuous, and by the Arzelà-Ascoli theorem, the operator $H: C([0, T], \mathbb{R}) \rightarrow C([0, T], \mathbb{R})$ is completely continuous.

In the last step we show that the operator $H$ has a fixed point. Let $H(x)=x$ be a solution. Then, for $t \in[0, T]$,

$$
\|H x\|=\|x\| \leq \Psi(\|x\|)\|\Omega\| \Phi,
$$

which implies that

$$
\frac{\|x\|}{\Psi(\|x\|)\|\Omega\| \Phi} \leq 1
$$

In view of $\left(S_{4}\right)$, there exists positive $M$ such that $\|x\| \neq M$. Let us set

$$
U=\{x \in C([0, T], \mathbb{R}):\|x\|<M\} .
$$

Then the operator $H: \bar{U} \rightarrow C([0, T], \mathbb{R})$ is continuous and completely continuous. From the choice of $U$, there is no $x \in \partial U$ such that $x=\mu H x$ for some $\mu \in(0,1)$. It can be proved by using contraction. Assume that there exists $x \in \partial U$ such that $x=\mu H x$ for some $\mu \in$ $(0,1)$. Then

$$
\begin{aligned}
& \|x\|=\|\mu H x\| \leq\|H x\| \leq \Psi(\|x\|)\|\Omega\| \Phi, \\
& \frac{\|x\|}{\Psi(\|x\|)\|\Omega\| \Phi} \leq 1 .
\end{aligned}
$$

This contradicts

$$
\frac{\|x\|}{\Psi(\|x\|)\|\Omega\| \Phi}>1
$$


Consequently, by the nonlinear alternative of Leray-Schauder type, we conclude that $H$ has a fixed point $x \in \bar{U}$, which is a solution of problem (1). This completes the proof.

Theorem 13 ([29]) Let $M$ be a closed, bounded, convex, and nonempty subset of a Banach space X. Let $A, B$ be the operators such that (a) $A x+B y \in M$ whenever $x, y \in M$; (b) $A$ is compact and continuous; (c) $B$ is a contraction mapping. Then there exists $z \in M$ such that $z=A z+B z$.

Theorem 14 Let $f:[0, T] \times \mathbb{R} \rightarrow \mathbb{R}$ be a continuous function, and let condition $\left(S_{1}\right)$ hold. In addition, the function $f$ satisfies the assumptions:

$\left(S_{5}\right)$ There exists a nonnegative function $\Omega \in C([0, T], \mathbb{R})$ such that

$$
|f(t, u)| \leq \Omega(t)
$$

$$
\text { for any }(t, u) \in[0, T] \times \mathbb{R} \text {. }
$$

$\left(S_{6}\right) L \Phi_{1}<1$, where $\Phi_{1}$ is defined by $(21)$.

Then the boundary value problem (1) has at least one solution on $[0, T]$.

Proof We first define the new operators $H_{1}$ and $H_{2}$ as

$$
\begin{aligned}
\left(H_{1} x\right)(t)= & J^{\alpha} f(s, x(s))(t)-\frac{1}{\varpi_{1}(\beta, \epsilon)} J^{\alpha} f(s, x(s))(T) \\
& +\frac{1}{\varpi_{1}(\gamma, \eta)}\left(\frac{\varpi_{2}(\beta, \epsilon)}{\varpi_{1}(\beta, \epsilon)}-t\right) J^{\alpha-1} f(s, x(s))(T) \\
& +\frac{1}{\varpi_{1}(\delta, \zeta)}\left(\frac{\varpi_{3}(\beta, \epsilon)}{2 \varpi_{1}(\beta, \epsilon)}-\frac{\varpi_{2}(\beta, \epsilon) \varpi_{2}(\gamma, \eta)}{\varpi_{1}(\beta, \epsilon) \varpi_{1}(\gamma, \eta)}\right. \\
& \left.+\frac{\varpi_{2}(\gamma, \eta) t}{\varpi_{1}(\gamma, \eta)}-\frac{t^{2}}{2}\right) J^{\alpha-2} f(s, x(s))(T)
\end{aligned}
$$

and

$$
\begin{aligned}
\left(H_{2} x\right)(t)= & \frac{\beta}{\varpi_{1}(\beta, \epsilon)}{ }^{\rho} I^{q} J^{\alpha} f(s, x(s))(\epsilon) \\
& -\frac{\gamma}{\varpi_{1}(\gamma, \eta)}\left(\frac{\varpi_{2}(\beta, \epsilon)}{\varpi_{1}(\beta, \epsilon)}-t\right){ }^{\rho} I^{q} J^{\alpha-1} f(s, x(s))(\eta) \\
& -\frac{\delta}{\varpi_{1}(\delta, \zeta)}\left(\frac{\varpi_{3}(\beta, \epsilon)}{2 \varpi_{1}(\beta, \epsilon)}-\frac{\varpi_{2}(\beta, \epsilon) \varpi_{2}(\gamma, \eta)}{\varpi_{1}(\beta, \epsilon) \varpi_{1}(\gamma, \eta)}\right. \\
& \left.+\frac{\varpi_{2}(\gamma, \eta) t}{\varpi_{1}(\gamma, \eta)}-\frac{t^{2}}{2}\right){ }^{\rho} I^{q} J^{\alpha-2} f(s, x(s))(\zeta) .
\end{aligned}
$$

Then we consider a closed, bounded, convex, and nonempty subset of the Banach space $X$ as

$$
B_{d}=\{x \in C:\|x\|<d\} \quad \text { with }\|\Omega\| \Phi \leq d,
$$

where $\Phi$ is defined by (20). Now, we show that $H_{1} x+H_{2} y \in B_{d}$ for any $x, y \in B_{d}$, where $H_{1}$ 
and $H_{2}$ are denoted by (23) and (24), respectively.

$$
\begin{aligned}
& \left\|H_{1} x+H_{2} y\right\| \leq J^{\alpha}|f(s, x(s))|(T) \\
& +\frac{1}{\left|\varpi_{1}(\beta, \epsilon)\right|}\left(J^{\alpha}|f(s, x(s))|(T)+|\beta|^{\rho} I^{q} J^{\alpha}|f(s, x(s))|(\epsilon)\right) \\
& +\frac{1}{\left|\varpi_{1}(\gamma, \eta)\right|}\left(\frac{\left|\varpi_{2}(\beta, \epsilon)\right|}{\left|\varpi_{1}(\beta, \epsilon)\right|}+T\right) \\
& \times\left(J^{\alpha-1}|f(s, x(s))|(T)+|\gamma|^{\rho} I^{q} J^{\alpha-1} f(s, x(s))(\eta)\right) \\
& +\frac{1}{\left|\varpi_{1}(\delta, \zeta)\right|}\left(\frac{\left|\varpi_{3}(\beta, \epsilon)\right|}{2\left|\varpi_{1}(\beta, \epsilon)\right|}+\frac{\left|\varpi_{2}(\beta, \epsilon)\right|\left|\varpi_{2}(\gamma, \eta)\right|}{\left|\varpi_{1}(\beta, \epsilon)\right|\left|\varpi_{1}(\gamma, \eta)\right|}\right. \\
& \left.+\frac{\left|\varpi_{2}(\gamma, \eta)\right| T}{\left|\varpi_{1}(\gamma, \eta)\right|}+\frac{T^{2}}{2}\right) \\
& \times\left(J^{\alpha-2}|f(s, x(s))|(T)+|\delta|^{\rho} I^{q} J^{\alpha-2}|f(s, x(s))|(\zeta)\right) \\
& \leq J^{\alpha} \Omega(s)(T) \\
& +\frac{1}{\left|\varpi_{1}(\beta, \epsilon)\right|}\left(J^{\alpha} \Omega(s)(T)+|\beta|^{\rho} I^{q} J^{\alpha} \Omega(s)(\epsilon)\right) \\
& +\frac{1}{\left|\varpi_{1}(\gamma, \eta)\right|}\left(\frac{\left|\varpi_{2}(\beta, \epsilon)\right|}{\left|\varpi_{1}(\beta, \epsilon)\right|}+T\right) \\
& \times\left(J^{\alpha-1} \Omega(s)(T)+|\gamma|^{\rho} I^{q} J^{\alpha-1} \Omega(s)(\eta)\right) \\
& +\frac{1}{\left|\varpi_{1}(\delta, \zeta)\right|}\left(\frac{\left|\varpi_{3}(\beta, \epsilon)\right|}{2\left|\varpi_{1}(\beta, \epsilon)\right|}+\frac{\left|\varpi_{2}(\beta, \epsilon)\right|\left|\varpi_{2}(\gamma, \eta)\right|}{\left|\varpi_{1}(\beta, \epsilon)\right|\left|\varpi_{1}(\gamma, \eta)\right|}\right. \\
& \left.+\frac{\left|\varpi_{2}(\gamma, \eta)\right| T}{\left|\varpi_{1}(\gamma, \eta)\right|}+\frac{T^{2}}{2}\right) \\
& \times\left(J^{\alpha-2} \Omega(s)(T)+|\delta|^{\rho} I^{q} J^{\alpha-2} \Omega(s)(\zeta)\right) \\
& \leq\|\Omega\|\left\{\frac{T^{\alpha}}{\Gamma(\alpha+1)}+\frac{1}{\left|\varpi_{1}(\beta, \epsilon)\right| \Gamma(\alpha+1)}\right. \\
& \times\left(T^{\alpha}+|\beta| \frac{\Gamma\left(\frac{\alpha+\rho}{\rho}\right)}{\Gamma\left(\frac{\alpha+\rho q+\rho}{\rho}\right)} \frac{\epsilon^{\alpha+\rho q}}{\rho^{q}}+\right) \\
& +\frac{1}{\left|\varpi_{1}(\gamma, \eta)\right| \Gamma(\alpha)}\left(\frac{\left|\varpi_{2}(\beta, \epsilon)\right|}{\left|\varpi_{1}(\beta, \epsilon)\right|}+T\right) \\
& \times\left(T^{\alpha-1}+|\gamma| \frac{\Gamma\left(\frac{\alpha-1+\rho}{\rho}\right)}{\Gamma\left(\frac{\alpha-1+\rho q+\rho}{\rho}\right)} \frac{\eta^{\alpha-1+\rho q}}{\rho^{q}}\right) \\
& +\frac{1}{\left|\varpi_{1}(\delta, \zeta)\right| \Gamma(\alpha-1)}\left(\frac{\left|\varpi_{3}(\beta, \epsilon)\right|}{2\left|\varpi_{1}(\beta, \epsilon)\right|}\right. \\
& \left.+\frac{\left|\varpi_{2}(\beta, \epsilon)\right|\left|\varpi_{2}(\gamma, \eta)\right|}{\left|\varpi_{1}(\beta, \epsilon)\right|\left|\varpi_{1}(\gamma, \eta)\right|}+\frac{\left|\varpi_{2}(\gamma, \eta)\right| T}{\left|\varpi_{1}(\gamma, \eta)\right|}+\frac{T^{2}}{2}\right) \\
& \left.\times\left(T^{\alpha-2}+|\delta| \frac{\Gamma\left(\frac{\alpha-2+\rho}{\rho}\right)}{\Gamma\left(\frac{\alpha-2+\rho q+\rho}{\rho}\right)} \frac{\zeta^{\alpha-2+\rho q}}{\rho^{q}}\right)\right\} \\
& =\|\Omega\| \Phi \leq d .
\end{aligned}
$$

Therefore, it is clear that $\left\|H_{1} x+H_{2} y\right\| \leq d$. Hence, $H_{1} x+H_{2} y \in B_{d}$. 
The next step is related to the compactness and continuity of the operator $H_{1}$. The proof is similar to that of Theorem 12.

Finally, we show that the operator $H_{2}$ is a contraction. By using assumption $\left(S_{1}\right)$,

$$
\begin{aligned}
& \left\|H_{2} x-H_{2} y\right\| \leq \frac{|\beta|}{\left|\varpi_{1}(\beta, \epsilon)\right|} \rho^{\rho} I^{q} J^{\alpha}|f(s, x(s))-f(s, y(s))|(\epsilon) \\
& +\frac{|\gamma|}{\left|\varpi_{1}(\gamma, \eta)\right|}\left(\frac{\left|\varpi_{2}(\beta, \epsilon)\right|}{\left|\varpi_{1}(\beta, \epsilon)\right|}+T\right) \\
& \times{ }^{\rho} I^{q} J^{\alpha-1}|f(s, x(s))-f(s, y(s))|(\eta) \\
& +\frac{|\delta|}{\left|\varpi_{1}(\delta, \zeta)\right|}\left(\frac{\left|\varpi_{3}(\beta, \epsilon)\right|}{2\left|\varpi_{1}(\beta, \epsilon)\right|}+\frac{\left|\varpi_{2}(\beta, \epsilon)\right|\left|\varpi_{2}(\gamma, \eta)\right|}{\left|\varpi_{1}(\beta, \epsilon)\right|\left|\varpi_{1}(\gamma, \eta)\right|}\right. \\
& \left.+\frac{\left|\varpi_{2}(\gamma, \eta)\right| T}{\left|\varpi_{1}(\gamma, \eta)\right|}+\frac{T^{2}}{2}\right) \\
& \times{ }^{\rho} I^{q} J^{\alpha-2}|f(s, x(s))-f(s, y(s))|(\zeta) \\
& \leq L\|x-y\|\left\{{\frac{|\beta|}{\left|\varpi_{1}(\beta, \epsilon)\right|}}^{\rho} I^{q} J^{\alpha}(1)(\epsilon)\right. \\
& +\frac{|\gamma|}{\left|\varpi_{1}(\gamma, \eta)\right|}\left(\frac{\left|\varpi_{2}(\beta, \epsilon)\right|}{\left|\varpi_{1}(\beta, \epsilon)\right|}+T\right){ }^{\rho} I^{q} J^{\alpha-1}(1)(\eta) \\
& +\frac{|\delta|}{\left|\varpi_{1}(\delta, \zeta)\right|}\left(\frac{\left|\varpi_{3}(\beta, \epsilon)\right|}{2\left|\varpi_{1}(\beta, \epsilon)\right|}+\frac{\left|\varpi_{2}(\beta, \epsilon)\right|\left|\varpi_{2}(\gamma, \eta)\right|}{\left|\varpi_{1}(\beta, \epsilon)\right|\left|\varpi_{1}(\gamma, \eta)\right|}\right. \\
& \left.\left.+\frac{\left|\varpi_{2}(\gamma, \eta)\right| T}{\left|\varpi_{1}(\gamma, \eta)\right|}+\frac{T^{2}}{2}\right){ }^{\rho} I^{q} J^{\alpha-2}(1)(\zeta)\right\} \\
& \leq L\left\{\frac{|\beta|}{\left|\varpi_{1}(\beta, \epsilon)\right| \Gamma(\alpha+1)} \frac{\Gamma\left(\frac{\alpha+\rho}{\rho}\right)}{\Gamma\left(\frac{\alpha+\rho q+\rho}{\rho}\right)} \frac{\epsilon^{\alpha+\rho q}}{\rho^{q}}\right. \\
& +\frac{|\gamma|}{\left|\varpi_{1}(\gamma, \eta)\right| \Gamma(\alpha)}\left(\frac{\left|\varpi_{2}(\beta, \epsilon)\right|}{\left|\varpi_{1}(\beta, \epsilon)\right|}+T\right) \\
& \times \frac{\Gamma\left(\frac{\alpha-1+\rho}{\rho}\right)}{\Gamma\left(\frac{\alpha-1+\rho q+\rho}{\rho}\right)} \frac{\eta^{\alpha-1+\rho q}}{\rho^{q}} \\
& +\frac{|\delta|}{\left|\varpi_{1}(\delta, \zeta)\right| \Gamma(\alpha-1)}\left(\frac{\left|\varpi_{3}(\beta, \epsilon)\right|}{2\left|\varpi_{1}(\beta, \epsilon)\right|}+\frac{\left|\varpi_{2}(\beta, \epsilon)\right|\left|\varpi_{2}(\gamma, \eta)\right|}{\left|\varpi_{1}(\beta, \epsilon)\right|\left|\varpi_{1}(\gamma, \eta)\right|}\right. \\
& \left.+\frac{\left|\varpi_{2}(\gamma, \eta)\right| T}{\left|\varpi_{1}(\gamma, \eta)\right|}+\frac{T^{2}}{2}\right) \\
& \left.\times \frac{\Gamma\left(\frac{\alpha-2+\rho}{\rho}\right)}{\Gamma\left(\frac{\alpha-2+\rho q+\rho}{\rho}\right)} \frac{\zeta^{\alpha-2+\rho q}}{\rho^{q}}\right\}\|x-y\| \\
& =L \Phi_{1}\|x-y\| \text {, }
\end{aligned}
$$

which means $\left\|H_{2} x-H_{2} y\right\| \leq L \Phi_{1}\|x-y\|$. As $L \Phi_{1}<1$, the operator $H_{2}$ is a contraction. For this reason, problem (1) has at least one solution on $[0, T]$.

\section{Examples}

In this section, some examples are illustrated to show our results. 
Example 1 Consider the following nonlinear fractional differential equation with Katugampola fractional integral conditions:

$$
\left\{\begin{array}{l}
{ }^{c} D^{5 / 2} x(t)=\frac{\sin ^{2}(\pi t)}{\left(e^{t}+10\right)}\left(\frac{|x(t)|}{|x(t)|+1}+1\right), \quad t \in\left[0, \frac{1}{2}\right] \\
x\left(\frac{1}{2}\right)=\frac{1}{2} I^{5} I^{\frac{1}{3}} x(3 / 8), \quad x^{\prime}\left(\frac{1}{2}\right)=\frac{1}{2}^{5} I^{\frac{1}{3}} x(1 / 3), \\
x^{\prime \prime}\left(\frac{1}{2}\right)=\frac{1}{2}^{5} I^{\frac{1}{3}} x(2 / 5) .
\end{array}\right.
$$

Here, $\alpha=5 / 2, T=\frac{1}{2}, \beta=1 / 2, \gamma=1 / 2, \delta=1 / 2, \epsilon=3 / 8, \eta=1 / 3, \zeta=2 / 5, \rho=5, q=\frac{1}{3}$, and

$$
f(t, x)=\frac{\sin ^{2}(\pi t)}{\left(e^{t}+10\right)}\left(\frac{|x|}{|x|+1}+1\right)
$$

Hence, we have $|f(t, x)-f(t, y)| \leq \frac{1}{10}\|x-y\|$. Then, assumption $\left(S_{1}\right)$ is satisfied with $L=\frac{1}{10}$. By using the Matlab program, $\omega_{1}\left(\frac{1}{2}, \frac{3}{8}\right)=0.9361, \omega_{1}\left(\frac{1}{2}, \frac{1}{3}\right)=0.9475, \omega_{1}\left(\frac{1}{2}, \frac{2}{5}\right)=0.9289$, $\omega_{2}\left(\frac{1}{2}, \frac{3}{8}\right)=0.4779, \omega_{2}\left(\frac{1}{2}, \frac{1}{3}\right)=0.4838, \omega_{3}\left(\frac{1}{2}, \frac{3}{8}\right)=0.4922$, and $\Phi=1.2261$ are found. Therefore, $L \Phi=0.1226<1$, which implies that assumption $\left(S_{2}\right)$ holds true. By using Theorem 10, the boundary value problem (25) has a unique solution on $\left[0, \frac{1}{2}\right]$.

Example 2 Consider the following nonlinear fractional differential equation with Katugampola fractional integral conditions:

$$
\left\{\begin{array}{l}
D^{5 / 2} x(t)=\left(\frac{t^{2}+1}{10}\right)\left(\frac{x^{2}(t)}{|x(t)|+1}+\frac{\sqrt{|x(t)|}}{2(1+\sqrt{|x(t)|}}+\frac{1}{2}\right), \quad t \in\left[0, \frac{1}{2}\right], \\
x\left(\frac{1}{2}\right)=\frac{1}{2}^{5} I^{\frac{1}{3}} x(3 / 8), \quad x^{\prime}\left(\frac{1}{2}\right)=\frac{1}{2}^{5} I^{\frac{1}{3}} x(1 / 3), \\
x^{\prime \prime}\left(\frac{1}{2}\right)=\frac{1}{2}^{5} I^{\frac{1}{3}} x(2 / 5),
\end{array}\right.
$$

where $\alpha=5 / 2, T=\frac{1}{2}, \beta=1 / 2, \gamma=1 / 2, \delta=1 / 2, \epsilon=3 / 8, \eta=1 / 3, \zeta=2 / 5, \rho=5, q=\frac{1}{3}$. Moreover,

$$
|f(t, u)|=\left|\left(\frac{t^{2}+1}{10}\right)\left(\frac{u^{2}}{|u|+1}+\frac{\sqrt{|u|}}{2(1+\sqrt{|u|})}+\frac{1}{2}\right)\right| \leq \frac{\left(t^{2}+1\right)(|u|+1)}{10} .
$$

By using assumption $\left(S_{3}\right)$, it is easy to see that $\Omega(t)=\frac{t^{2}+1}{10}$ and $\Psi(|u|)=|u|+1$. Moreover, $\|\Omega\|=\frac{1}{8}$ and $\Phi=1.2261$ which was found in the previous example. Now, we need to show that there exists $M>0$ such that

$$
\frac{M}{\Psi(M)\|\Omega\| \Phi}>1
$$

and such $M>0$ exists if

$$
1-\|\Omega\| \Phi>0
$$

By using direct computation $\|\Omega\| \Phi=0.1533<1$, assumption $\left(S_{4}\right)$ is satisfied. Hence, by using Theorem 12, the boundary value problem (26) has at least one solution on $\left[0, \frac{1}{2}\right]$. 
Example 3 Consider the following nonlinear fractional differential equation with Katugampola fractional integral conditions:

$$
\left\{\begin{array}{l}
{ }^{c} D^{5 / 2} x(t)=\frac{9 \sin ^{2}(\pi t)}{\left(e^{t}+10\right)}\left(\frac{|x(t)|}{|x(t)|+1}+1\right), \quad t \in\left[0, \frac{1}{2}\right], \\
x\left(\frac{1}{2}^{\prime}\right)=\frac{1}{2}^{5} I^{\frac{1}{3}} x(3 / 8), \quad x^{\prime}\left(\frac{1}{2}\right)=\frac{1}{2}^{5} I^{\frac{1}{3}} x(1 / 3), \\
x^{\prime \prime}\left(\frac{1}{2}\right)=\frac{1}{2}^{5} I^{\frac{1}{3}} x(2 / 5) .
\end{array}\right.
$$

Here, $\alpha=5 / 2, T=\frac{1}{2}, \beta=1 / 2, \gamma=1 / 2, \delta=1 / 2, \epsilon=3 / 8, \eta=1 / 3, \zeta=2 / 5, \rho=5, q=\frac{1}{3}$, and

$$
f(t, x)=\frac{9 \sin ^{2}(\pi t)}{\left(e^{t}+10\right)}\left(\frac{|x|}{|x|+1}+1\right) .
$$

Since $|f(t, x)-f(t, y)| \leq \frac{9}{10}|x-y|$, then it implies that $L=\frac{9}{10}$ means $\left(S_{1}\right)$ is satisfied but $\left(S_{2}\right)$, which is $L \Phi<1$, is not satisfied. [ $L \Phi=1.10358>1$.] Therefore, we consider $\left(S_{5}\right)$ which is

$$
|f(t, x)| \leq \frac{9}{\left(e^{t}+10\right)}\left(\frac{|x|}{|x|+1}+1\right) \leq \frac{18}{\left(e^{t}+10\right)}=\Omega(t) .
$$

By using (21), $\Phi_{1}=0.0561$ is found. It is obvious that $L \Phi_{1}=0.05049<1$. So, $\left(S_{6}\right)$ is satisfied. Hence, by using Theorem 14, the boundary value problem (27) has at least one solution on $\left[0, \frac{1}{2}\right]$.

\section{Acknowledgements}

The authors would like to thank the anonymous referees and the editor for their constructive suggestions on improving the presentation of the paper.

Competing interests

The authors declare that they have no competing interests.

\section{Authors' contributions}

All authors contributed equally to the writing of this paper. All authors read and approved the final manuscript.

\section{Publisher's Note}

Springer Nature remains neutral with regard to jurisdictional claims in published maps and institutional affiliations.

Received: 29 November 2017 Accepted: 25 February 2018 Published online: 05 March 2018

\section{References}

1. Kilbas, A.A., Srivastava, H.M., Trujillo, J.J.: Theory and Applications of Fractional Differential Equations. North-Holland Mathematics Studies, vol. 204. Elsevier, Amsterdam (2006)

2. Samko, S.G., Kilbas, A.A., Marichev, O.I.: Fractional Integrals and Derivatives: Theory and Applications. Gordon \& Breach, Yverdon (1993)

3. Bitsadze, A.V.: On the theory of nonlocal boundary value problems. Dokl. Akad. Nauk SSSR 277, 17-19 (1984)

4. Ahmad, B., Ntouyas, S.K., Tariboon, J.: Nonlocal fractional-order boundary value problems with generalized Riemann-Liouville integral boundary conditions. J. Comput. Anal. Appl. 23(7), 1281-1296 (2017)

5. Thongsalee, N., Ntouyas, S.K., Tariboon, J.: Nonlinear Riemann-Liouville fractional differential equations with nonlocal Erdelyi-Kober fractional integral conditions. Fract. Calc. Appl. Anal. 19(2), 480-497 (2016)

6. Ahmad, B.: Existence of solutions for fractional differential equations of order $q \in(2,3]$ with anti-periodic boundary conditions. J. Appl. Math. Comput. 34, 385-391 (2010)

7. Ahmad, B.: On nonlocal boundary value problems for nonlinear integro-differential equations of arbitrary fractional order. Results Math. 63, 183-194 (2013). https://doi.org/10.1007/s00025-011-0187-9

8. Ahmad, B., Alsaedi, A.: Existence and uniqueness of solutions for coupled systems of higher order nonlinear fractional differential equations. Fixed Point Theory Appl. 2010, Article ID 364560 (2010)

9. Ahmad, B., Nieto, J.J.: Existence results for nonlinear boundary value problems of fractional integro differential equations with integral boundary conditions. Bound. Value Probl. 2009, Article ID 708576 (2009)

10. Ntouyas, S.K.: Boundary value problems for nonlinear fractional differential equations and inclusions with nonlocal and fractional integral boundary conditions. Opusc. Math. 33, 117-138 (2013)

11. Ahmad, B., Nieto, J.J.: Existence results for a coupled system of nonlinear fractional differential equations with three-point boundary conditions. Comput. Math. Appl. 58, 1838-1843 (2009) 
12. Ahmad, B., Ntouyas, S.K., Alsaedi, A.: New existence results for nonlinear fractional differential equations with three-point integral boundary conditions. Adv. Differ. Equ. 2011, Article ID 107384 (2011)

13. Ahmad, B., Sivasundaram, S.: On four-point nonlocal boundary value problems of nonlinear integro-differential equations of fractional order. Appl. Math. Comput. 217, 480-487 (2010)

14. Bai, Z.B.: On positive solutions of a nonlocal fractional boundary value problem. Nonlinear Anal. 72, 916-924 (2010)

15. Balachandran, K., Trujillo, J.J.: The nonlocal Cauchy problem for nonlinear fractional integrodifferential equations in Banach spaces. Nonlinear Anal. 72, 4587-4593 (2010)

16. Benchohra, M., Hamani, S., Ntouyas, S.K.: Boundary value problems for differential equations with fractional order. Surv. Math. Appl. 3, 1-12 (2008)

17. Benchohra, M., Hamani, S., Ntouyas, S.K.: Boundary value problems for differential equations with fractional order and nonlocal conditions. Nonlinear Anal. 71, 2391-2396 (2009)

18. Bressan, A., Colombo, G.: Extensions and selections of maps with decomposable values. Stud. Math. 90, 69-86 (1988)

19. Byszewski, L., Lakshmikantham, V.: Theorem about the existence and uniqueness of a solution of a nonlocal abstract Cauchy problem in a Banach space. Appl. Anal. 40, 11-19 (1991)

20. Byszewski, L.: Theorems about existence and uniqueness of solutions of a semilinear evolution nonlocal Cauchy problem. J. Math. Anal. Appl. 162, 494-505 (1991)

21. Byszewski, L.: Existence and uniqueness of mild and classical solutions of semilinear functional-differential evolution nonlocal Cauchy problem. In: Selected Problems of Mathematics. 50th Anniv. Cracow Univ. Technol. Anniv. Issue, vol. 6, pp. 25-33. Cracow Univ. Technol., Krakow (1995)

22. Deimling, K.: Multivalued Differential Equations. de Gruyter, Berlin (1992)

23. Mahmudov, N., Unul, S.: Existence of solutions of fractional boundary value problems with $p$-Laplacian operator. Bound. Value Probl. 2015, 99 (2015)

24. Mahmudov, N., Awadalla, M., Abuassba, K.: Nonlinear sequential fractional differential equations with nonlocal boundary conditions. Adv. Differ. Equ. 2017, 319 (2017)

25. Zhang, L., Ahmad, B., Wang, G.: Existence and approximation of positive solutions for nonlinear fractional integro-differential boundary value problems on an unbounded domain. Appl. Comput. Math. 15, 149-158 (2016)

26. Guezane-Lakoud, A., Khaldi, R.: Solvability of a fractional boundary value problem with fractional integral condition. Nonlinear Anal. 75, 2692-2700 (2012)

27. Podlubny, I.: Fractional Differential Equations. Academic Press, New York (1999)

28. Katugampola, U.N.: New approach to a generalized fractional integral. Appl. Math. Comput. 218, 860-865 (2015)

29. Krasnoselskii, M.A.: Two remarks on the method of successive approximations. Usp. Mat. Nauk 10, 123-127 (1955)

\section{Submit your manuscript to a SpringerOpen ${ }^{\circ}$ journal and benefit from:}

- Convenient online submission

- Rigorous peer review

- Open access: articles freely available online

- High visibility within the field

- Retaining the copyright to your article

Submit your next manuscript at $>$ springeropen.com 